\title{
Bypass surgery versus medical treatment for symptomatic moyamoya disease in adults
}

\author{
Dong-Kyu Jang, MD, PhD, ${ }^{1}$ Kwan-Sung Lee, MD, PhD, ${ }^{2}$ Hyoung Kyun Rha, MD, PhD, ${ }^{3}$ \\ Pil-Woo Huh, MD, PhD, ${ }^{4}$ Ji-Ho Yang, MD, PhD, ${ }^{5}$ Ik Seong Park, MD, PhD, ${ }^{6}$ \\ Jae-Geun Ahn, MD, PhD, ${ }^{7}$ Jae Hoon Sung, MD, PhD, ${ }^{8}$ and Young-Min Han, MD, PhD ${ }^{1}$
}

\begin{abstract}
1Department of Neurosurgery, Incheon St. Mary's Hospital, College of Medicine, The Catholic University of Korea, Incheon; 2Department of Neurosurgery, Seoul St. Mary's Hospital, College of Medicine, The Catholic University of Korea, Seoul; ${ }^{3}$ Department of Neurosurgery, Youido St. Mary's Hospital, College of Medicine, The Catholic University of Korea, Seoul; ${ }^{4}$ Department of Neurosurgery, Uijeongbu St. Mary's Hospital, College of Medicine, The Catholic University of Korea, Uijeongbu; ${ }^{5}$ Department of Neurosurgery, Daejeon St. Mary's Hospital, College of Medicine, The Catholic University of Korea, Daejeon; ${ }^{6}$ Department of Neurosurgery, Bucheon St. Mary's Hospital, College of Medicine, The Catholic University of Korea, Bucheon; ${ }^{7}$ Department of Neurosurgery, St. Paul's Hospital, College of Medicine, The Catholic University of Korea, Seoul; and ${ }^{8}$ Department of Neurosurgery, St. Vincent's Hospital, College of Medicine, The Catholic University of Korea, Suwon, Korea
\end{abstract}

OBJECTIVE In this study the authors evaluated whether extracranial-intracranial bypass surgery can prevent stroke occurrence and decrease mortality in adult patients with symptomatic moyamoya disease (MMD).

METHODS The medical records of 249 consecutive adult patients with symptomatic MMD that was confirmed by digital subtraction angiography between 2002 and 2011 at 8 institutions were retrospectively reviewed. The study outcomes of stroke recurrence as a primary event and death during the 6 -year follow-up and perioperative complications within 30 days as secondary events were compared between the bypass and medical treatment groups.

RESULTS The bypass group comprised 158 (63.5\%) patients, and the medical treatment group comprised $91(36.5 \%)$ patients. For 249 adult patients with MMD, bypass surgery showed an HR of $0.48(95 \% \mathrm{Cl} 0.27-0.86, p=0.014)$ for stroke recurrence calculated by Cox regression analysis. However, for the 153 patients with ischemic MMD, the HR of bypass surgery for stroke recurrence was $1.07(95 \% \mathrm{Cl} 0.43-2.66, p=0.887)$. For the 96 patients with hemorrhagic $\mathrm{MMD}$, the multivariable adjusted $\mathrm{HR}$ of bypass surgery for stroke recurrence was $0.18(95 \% \mathrm{Cl} 0.06-0.49, \mathrm{p}=0.001)$. For the treatment modality, indirect bypass and direct bypass (or combined bypass) did not show any significant difference for stroke recurrence, perioperative stroke, or mortality (log rank; $p=0.524, p=0.828$, and $p=0.616$, respectively). CONCLUSIONS During the treatment of symptomatic MMD in adults, bypass surgery reduces stroke recurrence for the hemorrhagic type, but it does not do so for the ischemic type. The best choice of bypass methods in adult patients with MMD is uncertain. In adult ischemic MMD, a prospective randomized study to evaluate the effectiveness and safety of bypass surgery to prevent recurrent stroke is necessary.

https://thejns.org/doi/abs/10.3171/2016.8.JNS152875

KEY WORDS adult; moyamoya disease; bypass surgery; ischemic; hemorrhagic; vascular disorders

$\mathrm{M}$ OYAMOYa disease (MMD) is a chronic cerebrovascular disorder that is characterized by progressive stenosis or occlusion of the supraclinoid internal carotid artery (ICA) and its major branches in the vicinity of the circle of Willis. ${ }^{11}$ Many studies have investigated the epidemiological and clinical characteristics of
MMD. ${ }^{16,18,24,38}$ MMD in childhood usually presents with a transient ischemic attack (TIA) or cerebral infarction. More than half of adult patients with MMD present with cerebral hemorrhage. ${ }^{12,16}$ The clinical course and outcomes of surgical revascularization procedures differ substantially between children and adults. A recent meta-analysis of

ABBREVIATIONS DSA = digital subtraction angiography; ECA = external carotid artery; EDAGS = encephalo-duro-arterio-galeo-synangiosis; EDAMS = encephalo-duroarterio-myo-synangiosis; EDAS = encephalo-duro-arterio-synangiosis; EMS = encephalo-myo-synangiosis; ICA = internal carotid artery; JAM = Japanese Adult Moyamoya; $\mathrm{MCA}=$ middle cerebral artery; MMD = moyamoya disease; $\mathrm{mRS}$ = modified Rankin Scale; STA = superficial temporal artery; TIA = transient ischemic attack.

SUBMITTED December 8, 2015. ACCEPTED August 3, 2016.

INCLUDE WHEN CITING Published online November 11, 2016; DOI: 10.3171/2016.8.JNS152875. 
1448 pediatric MMD patients reported that 1156 (87\%) of them had symptomatic benefits after surgical revascularization achieved with either indirect or direct/combined bypass surgery. ${ }^{13}$ However, in adult MMD, the outcomes of long-term bypass surgery have rarely been compared with those of medical management.

The current study investigated the long-term treatment effect of bypass surgery for stroke prevention and mortality reduction in adult patients with symptomatic MMD in Korea in a real-world setting.

\section{Methods}

\section{Setting and Population}

For the current study, the subjects were drawn from consecutive patients treated at 8 hospitals between 2002 and 2011 who had a diagnosis of MMD confirmed by digital subtraction angiography (DSA) (ICD-10 code I67.5). This study was approved by the institutional review board of the Catholic Medical Center at the Catholic University of Korea. The inclusion criteria were as follows: 1) age $\geq$ 18 years; 2) MMD confirmed by DSA according to the diagnostic guidelines of MMD, as suggested by the Research Committee on Spontaneous Occlusion of the circle of Willis in 1997:11 3) newly confirmed MMD; and 4) Korean ethnicity. The exclusion criteria were incomplete radiological data, diagnosis or treatment at other institutions, and any treatment other than bypass surgery or medical management. This study was conducted as a retrospective cohort design, and the main weakness was a selection bias between bypass group and medical treatment group. The authors selected bypass candidates from study participants by assessing the degree of transdural anastomosis from the external carotid artery (ECA). The presence of transdural anastomosis from the ECA to more than 1 lobe was regarded as sufficient transdural anastomosis. Therefore, adult symptomatic MMD patients with insufficient transdural anastomosis from ECA were selected as bypass candidates. The patient screening process is displayed in Fig. 1. Among 884 candidates, 373 had DSA-confirmed MMD. Because the goal of this study was to determine the treatment effect of bypass surgery in adult symptomatic MMD, 88 patients were excluded due to asymptomatic MMD ( $=39)$, incomplete data $(n=43)$, aneurysm clipping $(n=1)$, endovascular treatment $(n=3)$, or stem cell injections during bypass $(\mathrm{n}=2)$. Among a total of 285 study participants, 249 patients were included in the final analysis; 33 patients were excluded due to sufficient transdural anastomosis, and data were unavailable for 3 .

\section{Classification and Baseline Data Acquisition}

All symptomatic MMD patients were classified as having one of 2 types of the disease according to symptom presentation: ischemic or hemorrhagic. Neurological symptoms included sudden severe headache, muscle weakness, sensory change, speech disturbance, visual disturbance, decreased consciousness, cognition impairment, and seizure. Patients who experienced a TIA or had a high signal lesion larger than $0.5 \mathrm{~cm}$ on diffusion-weighted images, T2-weighted MR images, or fluid-attenuated inversion recovery images, concurrent with acute neurological symptoms, were considered to have ischemic MMD. Cases of hemorrhagic MMD included those in which symptomatic intracranial hemorrhage was confirmed by CT or abnormal lesions with low signals, indicating a hemosiderin rim larger than $0.5 \mathrm{~cm}$, were observed on T2-weighted or gradient echo images concurrent with acute neurological symptoms. Asymptomatic MMD was defined as the absence of TIA and symptomatic stroke episodes. For patients who presented with both symptomatic ischemia and hemorrhage before diagnosis, the MMD type was determined according to the most recent stroke type. $\mathrm{Pa}$ tients with abnormal lesions on CT or MRI larger than 0.5 $\mathrm{cm}$ but no neurological symptoms were also considered

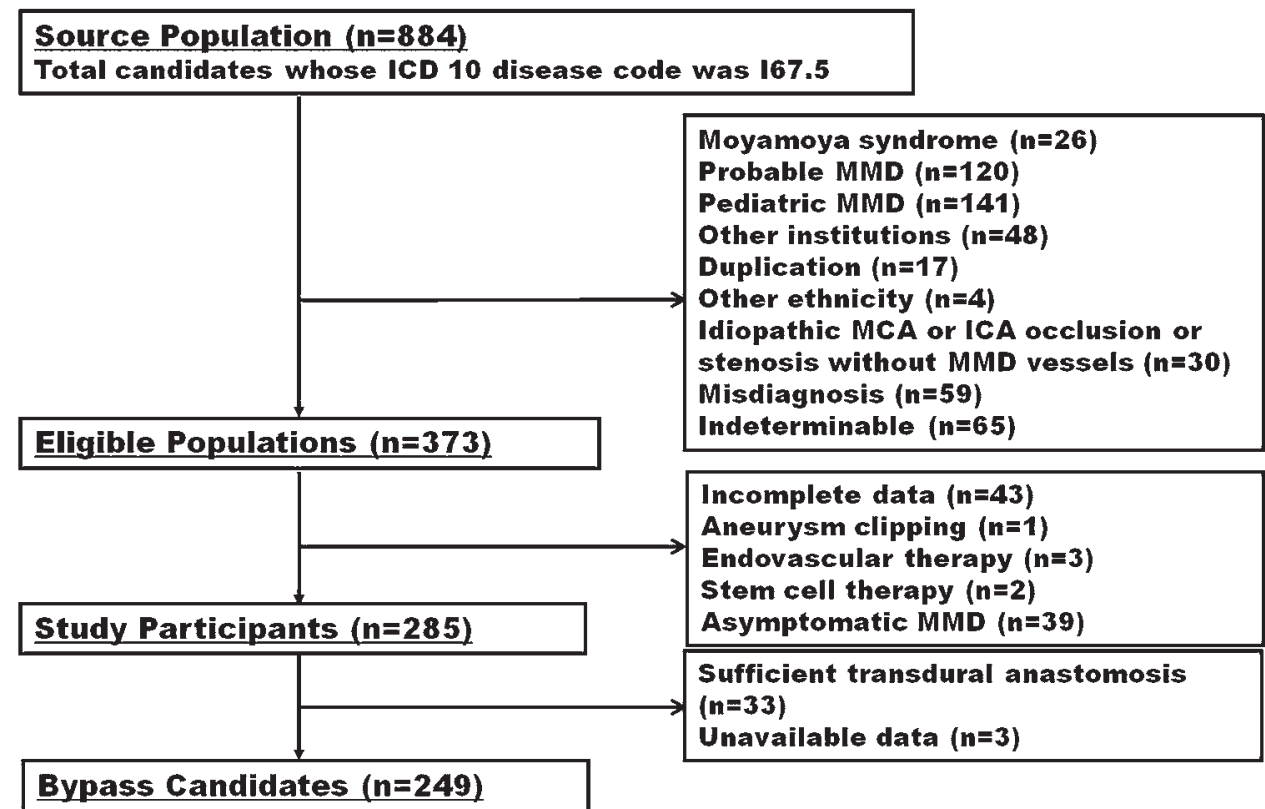

FIG. 1. Screening of adult symptomatic patients with definite MMD. 
to have asymptomatic MMD. Baseline characteristics, such as age, sex, family history of MMD, hypertension, diabetes, current smoking habit, heavy alcohol consumption ( $>60 \mathrm{~g}$ a day), hyperlipidemia, heart disease (atrial fibrillation, ischemic heart disease, and valvular heart disease), initial modified Rankin Scale (mRS) score, and angiographic stage of DSA, were also examined. ${ }^{37}$ Hypertension, diabetes, and hyperlipidemia were only indicated in patients who had received medication for more than 1 month or were receiving medication at discharge. Heart disease was only considered to be present if it was diagnosed by a cardiologist.

\section{Bypass Group and Medical Treatment Group}

Bypass surgery was chosen based on a thorough interview of the patient's legal representatives, and the indications for operation were at the discretion of each surgeon. All MMD patients were classified into 2 groups: bypass and medical treatment. The bypass surgery group was subdivided into direct and indirect bypass groups. The direct bypass group included patients who underwent superficial temporal artery-to-middle cerebral artery (STAMCA) anastomosis. The indirect bypass group included patients who underwent encephalo-duro-arterio-synangiosis (EDAS), encephalo-duro-arterio-galeo-synangiosis (EDAGS), encephalo-duro-arterio-myo-synangiosis (EDAMS), encephalo-myo-synangiosis (EMS), inverted EDAS, and inverted EDAGS. Patients who were treated with a combination of direct and indirect methods, termed combined bypass surgery, such as STA-MCA anastomosis with EDAS, STA-MCA anastomosis with EDAGS, and STA-MCA anastomosis with EDAMS, were classified into the direct bypass group. In patients treated with combined bypass surgery, the frontal branch of the STA served as the donor for the direct bypass, and the parietal branch was used for the indirect bypass. Patients who underwent direct bypass of at least 1 hemisphere were classified into the direct bypass group. Patients who received medical treatment for more than 1 year from the date of diagnosis before undergoing bypass surgery were classified into medical treatment group. Patients who underwent their first bypass surgery within 12 months of the date of diagnosis were classified into the bypass group. In addition, all patients who underwent bypass surgery of at least 1 hemisphere were classified into the bypass group.

\section{Outcome Measurements and End Points}

The primary event was defined as the occurrence of any stroke during the 6-year follow-up period. The censoring date was the date of loss to follow-up and termination of the current study period. In the medical treatment group, for patients who had been medically treated for more than 12 months after diagnosis and then underwent any type of bypass surgery, the censoring date was the date of the first bypass surgery. A new stroke was defined as the presentation of new neurological symptoms recorded by the site physicians and identifiable on CT or MR images or radiological findings recorded by a radiologist. The event dates were the dates of the development of stroke symptoms. If there was no medical record of stroke with abnormali-
TABLE 1. Baseline characteristics of 249 adult patients with symptomatic MMD

\begin{tabular}{|c|c|c|c|}
\hline Characteristics & $\begin{array}{l}\text { Bypass } \\
\text { Group } \\
(n=158)\end{array}$ & $\begin{array}{c}\text { Medical } \\
\text { Treatment } \\
\text { Group }(n=91)\end{array}$ & $\begin{array}{c}p \\
\text { Value }\end{array}$ \\
\hline Mean age $\pm S D$, yrs & $39.8 \pm 10.7$ & $44.2 \pm 12.6$ & 0.004 \\
\hline Female sex & $103(65.2)$ & $59(64.8)$ & 0.955 \\
\hline Family history of MMD & $1(0.6)$ & $1(1.1)$ & 1.0 \\
\hline Hypertension & $33(20.9)$ & $30(33.0)$ & 0.035 \\
\hline Diabetes & $15(9.5)$ & $10(11.0)$ & 0.705 \\
\hline Hyperlipidemia & $2(1.3)$ & $1(1.1)$ & 1.0 \\
\hline Current smoker & 0 & $2(2.2)$ & 0.133 \\
\hline Heavy alcohol drinker* & $1(0.6)$ & $2(2.2)$ & 0.556 \\
\hline Heart disease & $2(1.3)$ & 0 & 0.534 \\
\hline MMD type & & & $0.001 \dagger$ \\
\hline \multicolumn{4}{|l|}{ Ischemic } \\
\hline Infarct & $76(48.1)$ & $32(35.2)$ & \\
\hline TIA & $33(20.9)$ & $12(13.2)$ & \\
\hline \multicolumn{4}{|l|}{ Hemorrhagic } \\
\hline $\mathrm{ICH}$ or IVH & $49(31.0)$ & $45(49.5)$ & \\
\hline $\mathrm{SAH}$ & 0 & $2(2.2)$ & \\
\hline Suzuki \& Takaku stage & & & 0.474 \\
\hline 1 & $4(2.5)$ & 0 & \\
\hline 2 & $36(22.8)$ & $27(29.7)$ & \\
\hline 3 & $73(46.2)$ & $35(38.5)$ & \\
\hline 4 & $30(19.0)$ & $18(19.8)$ & \\
\hline 5 & $13(8.2)$ & $10(11.0)$ & \\
\hline 6 & $2(1.3)$ & $1(1.1)$ & \\
\hline mRS Score $3-5$ at admission & $49(31.0)$ & $32(35.2)$ & 0.501 \\
\hline Antiplatelet medication $\ddagger$ & $117(74.1)$ & $42(46.2)$ & $<0.001$ \\
\hline \multicolumn{4}{|l|}{ Operated hemispheres } \\
\hline Unilateral & $104(65.8)$ & 0 & \\
\hline Direct & $53(33.5)$ & & \\
\hline Indirect & $51(32.3)$ & & \\
\hline Bilateral & $54(34.2)$ & 0 & \\
\hline Direct & $19(12.0)$ & & \\
\hline Indirect & $25(15.8)$ & & \\
\hline Mixed§ & $10(6.3)$ & & \\
\hline
\end{tabular}

$\mathrm{ICH}=$ intracerebral hemorrhage; $\mathrm{IVH}=$ intraventricular hemorrhage; $\mathrm{SAH}=$ subarachnoid hemorrhage.

Values are presented as the number of patients (\%) unless indicated otherwise.

* Daily alcohol consumption $>60 \mathrm{~g}$.

$\dagger$ The chi-square test was used to compare ischemic MMD and hemorrhagic $M M D$ according to treatment modality.

$\ddagger$ Aspirin (100 mg) or clopidogrel $(75 \mathrm{mg}$ ) or cilostazol (200 mg) was administered.

$\S$ Direct bypass in one hemisphere and indirect bypass in the other hemisphere were performed.

ties on CT or MR images, the patient was assumed to not have experienced any new events through the last followup date. Secondary outcomes such as death, and any perioperative complications, such as stroke or major adverse events that required a rescue operation within 30 days after every bypass surgery, were recorded. All periopera- 
TABLE 2. Summary of postoperative complications within 30 days in 158 patients treated with bypass surgery

\begin{tabular}{cccc}
\hline Postop Complication & $\begin{array}{c}\text { Ischemic MMD } \\
(n=109)\end{array}$ & $\begin{array}{c}\text { Hemorrhagic } \\
\text { MMD }(n=49)\end{array}$ & $\begin{array}{c}\mathrm{p} \\
\text { Value }\end{array}$ \\
\hline Stroke w/in 30 days (\%) & $18(16.5)$ & $1(2.0)$ & 0.008 \\
\hline Ischemic stroke & $17(15.6)$ & $1(2.0)$ & \\
\hline Hemorrhagic stroke & $1(0.9)$ & 0 & \\
\hline $\begin{array}{l}\text { Mechanical complications } \\
\text { (\%) }\end{array}$ & $4(3.7)$ & $4(8.2)$ & 0.255 \\
\hline SDH & 1 & 2 & \\
\hline Wound problem & 3 & 1 & \\
\hline Hydrocephalus & 0 & 1 & \\
\hline
\end{tabular}

$\mathrm{SDH}=$ subdural hemorrhage.

tive strokes were considered primary events. All data were validated by 2 investigators while they were being gathered and handled, and any disagreements between the 2 investigators with regard to the clinical and radiological measurements were resolved through discussion.

\section{Statistical Analysis}

Categorical variables were analyzed using the chisquare or Fisher exact test, and the t-test for continuous variables was used to examine age according to treatment. The Kaplan-Meier method was used to create the timeto-event curves. The log-rank test to compare constant hazard functions for events during the 6-year follow-up period was performed according to the treatment modality and bypass method. To calculate the hazard ratios of bypass surgery for stroke and death during the 6-year follow-up period and perioperative stroke within 30 days, a Cox proportional hazards model was used. To compare the efficacy of direct bypass versus indirect bypass, the log-rank test and Cox regression analysis for cumulative stroke incidence between direct bypass and indirect bypass were performed in only 148 patients with MMD who had undergone direct or indirect bypass surgery, excluding 10 patients treated with mixed bypass methods in both hemispheres (Table 1). Only variables with a $\mathrm{p}$ value $<0.05$ in the univariate Cox regression analysis were entered into the multivariate Cox proportional hazards model. For all statistical analyses, 2-tailed tests were used, and all probability values were uncorrected for multiple comparisons.
Significance was accepted if the $\mathrm{p}$ value was $<0.05$. All statistical computations were performed using SAS (version 9.2, SAS Institute Inc.).

\section{Results \\ Patient Characteristics}

The median age of the 249 adult symptomatic MMD patients was 41.7 years (interquartile range 32.8-49.7 years), and the male-to-female ratio was 1:1.86. Two patients had a positive family history for MMD $(0.7 \%)$. The presenting symptoms included TIA in 72 patients (28.9\%), decreased consciousness in $61(24.5 \%)$, speech disturbances in $45(18.1 \%)$, headaches in $18(7.2 \%)$, motor weakness in 37 (14.9\%), visual disturbances in 8 (3.2\%), sensory changes in $6(2.4 \%)$, seizures in $1(0.4 \%)$, and memory impairment in $1(0.4 \%)$. The initial $\mathrm{mRS}$ was scored as 0 in 129 patients (51.8\%), 1 in 17 (6.8\%), 2 in 22 (8.8\%), 3 in 31 (12.4\%), 4 in $26(10.4 \%)$, and 5 in 24 (9.6\%). Sixty-three patients $(25.3 \%)$ had comorbid hypertension, 25 (10.0\%) had diabetes, $3(1.2 \%)$ had hyperlipidemia, $2(0.8 \%)$ were current smokers, $3(1.2 \%)$ were heavy alcohol drinkers, and $2(0.8 \%)$ had heart disease.

Table 1 shows the comparison of the baseline characteristics between the bypass and medical treatment groups. The mean age was higher in the medical treatment group than in the bypass group $(\mathrm{p}=0.004)$. The distribution of ischemic MMD and hemorrhagic MMD was significantly different between the bypass and medical treatment groups $(\mathrm{p}=0.001)($ Table 1$)$.

\section{Radiological Profiles}

The angiographic staging according to the system of Suzuki and Takaku ${ }^{37}$ of the 249 patients revealed Stage 1 in 4 (1.6\%), Stage 2 in 63 (25.3\%), Stage 3 in 108 (43.4\%), Stage 4 in 48 (19.3\%), Stage 5 in $23(9.2 \%)$, and Stage 6 in $3(1.2 \%)$ patients. Regarding presentation type, 153 patients $(61.4 \%)$ had ischemic disease, and 96 patients (38.6\%) had hemorrhagic disease. Among the 153 ischemic MMD patients, 108 (70.6\%) presented with acute infarction, and 45 (29.4\%) presented with TIA. Among the 96 patients with hemorrhagic MMD, 94 (97.9\%) presented with intracerebral hemorrhage or intraventricular hemorrhage, and 2 (2.1\%) presented with subarachnoid hemorrhage. The distributions of the initial stroke subtypes were significantly different between the bypass and medical

TABLE 3. Summary of stroke recurrence and death according to treatment during a 6 -year follow-up of 249 adult patients

\begin{tabular}{|c|c|c|c|c|c|c|}
\hline \multirow[b]{2}{*}{ End Point } & \multicolumn{3}{|c|}{ Ischemic MMD } & \multicolumn{3}{|c|}{ Hemorrhagic MMD } \\
\hline & $\begin{array}{l}\text { Bypass Surgery } \\
\qquad(n=109)\end{array}$ & $\begin{array}{l}\text { Medical Treatment } \\
\qquad(\mathrm{n}=44)\end{array}$ & $\begin{array}{c}p \\
\text { Value }\end{array}$ & $\begin{array}{l}\text { Bypass Surgery } \\
\qquad(n=49)\end{array}$ & $\begin{array}{l}\text { Medical Treatment } \\
\qquad(\mathrm{n}=47)\end{array}$ & $\begin{array}{c}p \\
\text { Value }\end{array}$ \\
\hline Primary outcome & & & 0.073 & & & 0.008 \\
\hline Recurrent ischemic stroke & $19(17.4)$ & $3(6.8)$ & & $2(4.1)$ & $10(21.3)$ & \\
\hline Recurrent hemorrhagic stroke & $2(1.8)$ & $3(6.8)$ & & $3(6.1)$ & $7(14.9)$ & \\
\hline Secondary outcome & & & & & & 0.515 \\
\hline Death due to recurrent hemorrhage & 0 & 0 & & $3(6.1)$ & $3(6.4)$ & \\
\hline Death due to initial hemorrhage & 0 & 0 & & 0 & $2(4.3)$ & \\
\hline
\end{tabular}

Percentages, in parentheses, are based on the number of patients, rather than hemispheres, in each subgroup. 
treatment groups $(\mathrm{p}=0.001)$ (Table 1$)$. The distributions of the Suzuki and Takaku angiographic stages were not significantly different between the bypass and medical treatment groups $(\mathrm{p}=0.474)$ (Table 1$)$.

\section{Bypass Surgery and Perioperative Complications}

A total of 158 patients underwent bypass surgery in 212 hemispheres. Fifty-six (36.1\%) patients underwent surgical treatment in both hemispheres, and 104 (65.8\%) patients underwent surgery in 1 hemisphere. Seventy-two (45.6\%) patients were treated with direct bypass, and 76 (48.1\%) patients were treated with indirect bypass. Ten (6.3\%) patients were treated with mixed methods of direct bypass in one hemisphere and indirect bypass in the other. Among the 212 bypass procedures, STA-MCA anastomosis was performed in 52 hemispheres (24.5\%), EDAS in 71 $(33.5 \%)$, EDAGS in $25(11.8 \%)$, EMS in $1(0.5 \%)$, inverted EDAS in $5(2.4 \%)$, inverted EDAGS in $11(5.2 \%)$, STAMCA anastomosis with EDAS in 24 (11.3\%), and STAMCA anastomosis with EDAGS in 23 (10.8\%). Twentyseven (17.1\%) perioperative complications occurred within 30 days after the 212 bypass surgeries, including ischemic stroke in 17 patients (8.0\%), hemorrhagic stroke in 1 patient $(0.9 \%)$, subdural hematoma in 3 patients (1.4\%), wound problems in 4 patients $(1.9 \%)$, and hydrocephalus in 1 patient $(0.5 \%)$ (Table 2$)$.

\section{Follow-Up and Study Outcomes}

The median follow-up time for the 249 patients was 2.32 years (interquartile range $1.01-4.77$ years). Table 3 summarizes the primary and secondary events. During the 6-year follow-up period, 49 new strokes (34 infarcts and 15 hemorrhages) and 8 deaths occurred. The cause of death was recurrent hemorrhagic stroke in 6 patients and initial hemorrhagic stroke in 2 patients. In the bypass group, the primary events occurred in 26 of 158 patients (16.5\%); among the 109 patients with ischemic MMD, there were $19(17.4 \%)$ ischemic strokes and $2(1.8 \%)$ hemorrhagic strokes, and among the 49 patients with hemorrhagic MMD, there were $2(4.1 \%)$ ischemic strokes and $3(6.1 \%)$ hemorrhagic strokes (Table 3). However, in the medical treatment group, the recurrent strokes occurred in 23 of 91 patients (25.3\%); among the 44 patients with ischemic MMD, there were 3 ischemic strokes $(6.8 \%)$ and 3 hemorrhagic strokes (6.8\%), and among 47 patients hemorrhagic MMD patients, there were 10 ischemic strokes $(21.3 \%)$ and 7 hemorrhagic strokes (14.9\%). The overall incidence density of the primary events was 84.6 per 1000 person-years for all 249 adult patients with symptomatic MMD: 56.8 per 1000 person-years for the bypass group and 189.3 per 1000 person-years for the medical treatment group. For the patients with ischemic MMD, the overall incidence density of the primary events was 68.3 per 1000 person-years in the bypass group and 91.0 per 1000 person-years in the medical treatment group. In contrast, for the patients with hemorrhagic MMD, the overall incidence density of the primary events was 33.2 per 1000 personyears in the bypass group and 306.1 per 1000 person-years in the medical treatment group. For the 249 patients with symptomatic MMD, the overall incidence density of death
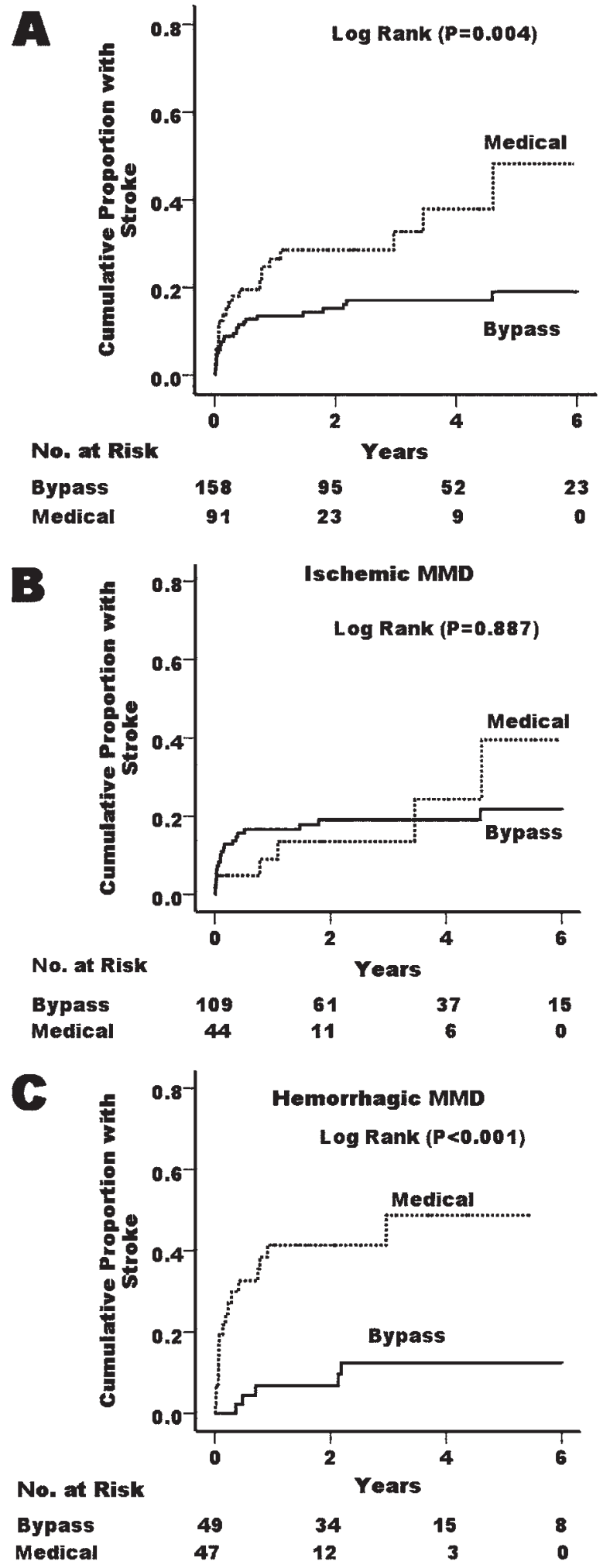

FIG. 2. Kaplan-Meier cumulative curves for stroke recurrence in 249 adult patients with MMD (A), 153 patients with ischemic MMD (B), and 96 hemorrhagic MMD patients (C). 
TABLE 4. Univariate and multivariate analyses for factors associated with stroke recurrence in 249 adult patients with symptomatic MMD

\begin{tabular}{|c|c|c|c|c|}
\hline \multirow[b]{2}{*}{ Variable } & \multicolumn{4}{|c|}{ Stroke Recurrence } \\
\hline & Univariate $^{*}$ & $p$ Value & Multivariate* & $\mathrm{p}$ Value \\
\hline \multicolumn{5}{|l|}{ Surgery status } \\
\hline Medical & 1.00 (reference) & & & \\
\hline Bypass & $0.45(0.25-0.79)$ & 0.005 & $0.48(0.27-0.86)$ & 0.014 \\
\hline Age (continuous) & $0.99(0.97-1.02)$ & 0.725 & & \\
\hline Age (>42 yrs, median) & $0.94(0.54-1.65)$ & 0.833 & & \\
\hline Female & $1.24(0.67-2.27)$ & 0.491 & & \\
\hline Family history & NA & & & \\
\hline Hypertension & $1.04(0.55-1.96)$ & 0.909 & & \\
\hline Diabetes & $0.51(0.16-1.65)$ & 0.261 & & \\
\hline Hyperlipidemia & NA & & & \\
\hline Current smoker & $23.85(5.34-106.55)$ & $<0.001$ & $15.65(3.41-71.76)$ & $<0.001$ \\
\hline Heavy alcohol drinker & $2.13(0.29-15.49)$ & 0.454 & & \\
\hline Heart disease & NA & & & \\
\hline \multicolumn{5}{|l|}{ MMD type } \\
\hline Ischemic & 1.00 (reference) & & & \\
\hline Hemorrhagic & $1.37(0.78-2.41)$ & 0.275 & & \\
\hline mRS Score $3-5$ at diagnosis & $1.44(0.81-2.54)$ & 0.212 & & \\
\hline \multicolumn{5}{|l|}{ Suzuki \& Takaku stage } \\
\hline 1 & 1.00 (reference) & & & \\
\hline 2 & $0.48(0.11-2.10)$ & 0.328 & & \\
\hline 3 & $0.32(0.07-1.38)$ & 0.126 & & \\
\hline 4 & $0.44(0.09-2.04)$ & 0.293 & & \\
\hline 5 & $0.69(0.14-3.41)$ & 0.646 & & \\
\hline 6 & $1.15(0.10-12.76)$ & 0.908 & & \\
\hline Antiplatelet agent & $0.84(0.47-1.50)$ & 0.550 & & \\
\hline
\end{tabular}

was 11.6 per 1000 person-years for all adult symptomatic MMD patients, 5.7 per 1000 person-years in the bypass group, and 30.5 per 1000 person-years in the medical treatment group.

Regarding the primary end point of stroke recurrence over the 6-year follow-up period in 249 adult MMD patients, the bypass group showed proportional cumulative curves for stroke incidence compared with the medical group (log-rank test, $\mathrm{p}=0.004$ ) (Fig. 2A). In 96 patients with hemorrhagic MMD, the Kaplan-Meier cumulative curves for the stroke recurrence showed a significant difference between the bypass and medical treatment groups (log-rank test, p < 0.001) (Fig. 2C). However, in 153 patients with ischemic MMD, the bypass and medical treatment groups showed nonproportional cumulative curves for stroke incidence (log-rank test, $\mathrm{p}=0.887$ ) (Fig. 2B). In the multivariate analysis for stroke recurrence in 249 adult patients with MMD, bypass surgery showed a preventive effect with an HR of 0.48 (95\% CI 0.27-0.86, p $=0.014$ ), and current smoking status was a strong predictor for stroke recurrence with an HR of 15.65 (95\% CI $3.41-71.76, \mathrm{p}<0.001$ ) (Table 4). In the 153 patients with ischemic MMD, the univariate HR for stroke recurrence of bypass surgery was $1.07(95 \%$ CI $0.43-2.66, p=0.887)$
(Table 5); on the contrary, in 96 patients with hemorrhagic MMD, the multivariate adjusted HR for stroke recurrence was 0.18 (95\% CI 0.06-0.49, p = 0.001) (Table 6).

The Kaplan-Meier cumulative curves for death due to recurrent stroke in 249 adult symptomatic MMD patients did not demonstrate any significant difference between the bypass and medical treatment groups (log-rank test, $\mathrm{p}=$ 0.279 ) (Fig. 3A). There was also no significant difference in the cumulative curves for recurrent stroke-related death between the bypass and medical treatment groups in the subgroup analysis of hemorrhagic MMD (log-rank test, $p$ $=0.716$ ) (Fig. 3B). In 148 patients with MMD treated with either direct bypass or indirect bypass, the cumulative hazard function for stroke recurrence was not significantly different between direct bypass and indirect bypass on 6-year follow-up and within 30 days $(\log$ rank, $\mathrm{p}=0.524$ and $\mathrm{p}=$ 0.828 , respectively) (Fig. $4 \mathrm{~A}$ and $\mathrm{B}$ ). There was no significant difference in the mortality rate between the direct bypass and indirect bypass surgery groups (Fig. 4C). In the univariate Cox regression analysis of 148 adult symptomatic MMD patients treated with bypass surgery, the HR of direct bypass surgery compared with indirect bypass surgery for perioperative stroke within 30 days during or following bypass surgery was 0.90 (95\% CI 0.35-2.34, p 
TABLE 5. Univariate and multivariate analyses for factors associated with stroke recurrence in 153 adult patients with ischemic MMD

\begin{tabular}{|c|c|c|c|c|}
\hline \multirow[b]{2}{*}{ Variable } & \multicolumn{4}{|c|}{ Stroke recurrence (HR with $95 \% \mathrm{Cl}, \mathrm{n}=153$ ) } \\
\hline & Univariate* $^{*}$ & p Value & Multivariate* $^{*}$ & $\mathrm{p}$ Value \\
\hline \multicolumn{5}{|l|}{ Surgery status } \\
\hline Medical & 1.00 (Reference) & & & \\
\hline Bypass & $1.07(0.43-2.66)$ & 0.887 & & \\
\hline Age (continuous) & $1.004(0.97-1.04)$ & 0.803 & & \\
\hline Age (>42 yrs, median) & $1.12(0.53-2.40)$ & 0.766 & & \\
\hline Female sex & $0.91(0.41-1.98)$ & 0.803 & & \\
\hline Family history & NA & & & \\
\hline Hypertension & $1.09(0.47-2.48)$ & 0.847 & & \\
\hline Diabetes & $0.74(0.22-2.46)$ & 0.623 & & \\
\hline Hyperlipidemia & NA & & & \\
\hline Current smoker & $29.22(3.41-250.16)$ & 0.002 & $29.22(3.41-250.16)$ & 0.002 \\
\hline Heavy alcohol drinker & $29.22(3.41-250.16)$ & 0.002 & NA & \\
\hline Heart disease & NA & & & \\
\hline $\mathrm{mRS}$ Score $3-5$ at diagnosis & $1.14(0.46-2.82)$ & 0.779 & & \\
\hline \multicolumn{5}{|l|}{ Suzuki \& Takaku stage } \\
\hline 1 & 1.00 (Reference) & & & \\
\hline 2 & $0.57(0.07-4.56)$ & 0.600 & & \\
\hline 3 & $0.38(0.05-3.07)$ & 0.364 & & \\
\hline 4 & $0.37(0.04-3.58)$ & 0.390 & & \\
\hline 5 & $1.11(0.13-9.55)$ & 0.924 & & \\
\hline 6 & $1.90(0.12-30.55)$ & 0.649 & & \\
\hline Antiplatelet agent & $2.42(0.33-17.89)$ & 0.386 & & \\
\hline
\end{tabular}

* Presented as $\mathrm{HR}(95 \% \mathrm{Cl})$.

$=0.828$, while that for the ischemic MMD type was 7.79 $(95 \%$ CI $1.02-58.09, \mathrm{p}=0.048)($ Table 7$)$.

\section{Discussion}

We demonstrated that bypass surgery prevented stroke recurrence in adult patients with hemorrhagic MMD but did not do so for adult patients with ischemic MMD. This study also did not confirm the effectiveness of bypass surgery on mortality reduction.

Until recently, bypass surgery has been preferentially performed over medical treatment in adults with ischemic MMD, while medical treatment has been preferentially performed over bypass surgery in adults with hemorrhagic MMD. ${ }^{5,6,16,27}$ Additionally, in this long-term cohort study, bypass surgery was performed more frequently in patients with ischemic MMD than in those with hemorrhagic MMD ( $p=0.001$, Table 1). This trend may be attributed to the lack of evidence of the benefit of bypass surgery in adult hemorrhagic MMD. Although many studies have reported the effectiveness of bypass surgery in adult MMD, ${ }^{4,9,16,17,25,31,34,35}$ only 1 randomized clinical trial showed a marginally preventive effect of direct bypass on subsequent bleeding in hemorrhagic MMD in adults. ${ }^{29}$ Some investigators have reported favorable results of bypass surgery on secondary stroke prevention in adult MMD, ${ }^{1,26,28,35}$ but others found no significant differences in secondary stroke prevention between surgical revascularization and medical management. ${ }^{9}$
In this study, bypass surgery to prevent stroke showed different results for ischemic and hemorrhagic MMD (Fig. 2, Tables 5 and 6). The bypass benefit (HR 0.18, 95\% CI 0.06-0.49) (Table 6) for recurrent stroke in our study was comparable to that of the Japanese Adult Moyamoya (JAM) trial of hemorrhage-onset MMD in adults (HR 0.355 , 95\% CI $0.125-1.009$ ). ${ }^{29}$ The finding of 5 rehemorrhages and 1 ischemic stroke in 42 patients in the bypass group of the JAM trial ${ }^{29}$ and 3 hemorrhages and 2 ischemic strokes in 49 patients in the bypass group in this study are encouraging (Table 3 ). Unlike previous observational studies of bypass surgery in hemorrhagic MMD in adults, ${ }^{17,20}$ our study and a recent large Chinese cohort study ${ }^{27}$ confirmed the preventive effect of bypass surgery on rebleeding in hemorrhagic MMD in a real-world setting. Therefore, in adult patients with hemorrhagic MMD, bypass surgery can reduce stroke incidence. Although many researchers have reported the benefits of bypass surgery in adult ischemic MMD, ${ }^{15,35}$ no randomized controlled trial has been performed in this population. In contrast to previous studies,,$^{5,14,15,28,35}$ we demonstrated no preventive effects for stroke throughout the postoperative period in adult patients with ischemic MMD (Fig. 2B and Table 5). However, the perioperative stroke rate of $9.0 \%$ (19 per 212 procedures) and the clinical course of disease observed here are similar to those in previous studies (3.0\%-13.3\%). . $^{3-5,7,19,28,35}$ Cho et al. reported 10 (13.0\%) perioperative infarctions and $2(2.6 \%)$ hemorrhagic events 
TABLE 6. Univariate and multivariate analyses for factors associated with stroke recurrence in 96 adult patients with hemorrhagic MMD

\begin{tabular}{|c|c|c|c|c|}
\hline \multirow[b]{2}{*}{ Variable } & \multicolumn{4}{|c|}{ Stroke Recurrence } \\
\hline & Univariate* $^{*}$ & p Value & Multivariate* & $p$ Value \\
\hline \multicolumn{5}{|l|}{ Surgery status } \\
\hline Medical & 1.00 (reference) & & & \\
\hline Bypass & $0.17(0.06-0.47)$ & 0.001 & $0.18(0.06-0.49)$ & 0.001 \\
\hline Age (continuous) & $0.98(0.95-1.02)$ & 0.327 & & \\
\hline Age (>42 yrs, median) & $0.71(0.30-1.63)$ & 0.415 & & \\
\hline Female & $1.89(0.70-5.13)$ & 0.211 & & \\
\hline Family history & NA & & & \\
\hline Hypertension & $1.03(0.38-2.79)$ & 0.957 & & \\
\hline Diabetes & NA & & & \\
\hline Hyperlipidemia & NA & & & \\
\hline Current smoker & $22.22(2.48-198.85)$ & 0.006 & $12.11(1.34-109.33)$ & 0.026 \\
\hline Heavy alcohol drinker & NA & & & \\
\hline Heart disease & NA & & & \\
\hline $\mathrm{mRS}$ Score $3-5$ at diagnosis & $1.56(0.66-3.73)$ & 0.313 & & \\
\hline \multicolumn{5}{|l|}{ Suzuki \& Takaku stage } \\
\hline 1 & 1.00 (reference) & & & \\
\hline 2 & $0.37(0.04-3.21)$ & 0.368 & & \\
\hline 3 & $0.20(0.03-1.63)$ & 0.133 & & \\
\hline 4 & $0.35(0.04-2.89)$ & 0.327 & & \\
\hline 5 & $0.21(0.01-3.34)$ & 0.267 & & \\
\hline 6 & NA & & & \\
\hline Antiplatelet agent & $0.78(0.29-2.13)$ & 0.633 & & \\
\hline
\end{tabular}

* Presented as HR $(95 \% \mathrm{Cl})$.

among 77 bypass surgeries in their study with thorough follow-up results for 60 adults with MMD; only 2 (2.6\%) hemorrhagic events and 1 (1.3\%) infarction occurred during the follow-up period. ${ }^{5}$ Because most previous studies defined perioperative stroke differently according to their definition of the perioperative period and duration or reversibility of symptoms, it is difficult to directly compare their postoperative stroke rates with ours. However, it appears that perioperative stroke risk may be relatively high in adult patients with ischemic MMD compared with those with hemorrhagic MMD. This phenomenon is similar to the results of extracranial-intracranial bypass surgery for stroke prevention obtained in a hemodynamic cerebral ischemia COSS (Carotid Occlusion Surgery Study) trial. ${ }^{33}$ That trial reported a perioperative stroke rate of $15 \%$ and a post-perioperative stroke risk of $6 \% .^{33}$

The observation of a higher perioperative stroke risk in adult patients with ischemic MMD relative to that in adult patients with hemorrhagic MMD may be due to hemodynamic differences between the 2 MMD types. ${ }^{30,32}$ In a PET study of patients with MMD, Nariai et al. demonstrated that cerebral blood flow, cerebral blood volume, and oxygen extraction fraction were significantly different between the hemorrhagic onset group and the group with TIA or infarction..$^{30}$ Our study did not measure preoperative and postoperative hemodynamic parameters, but we did observe an HR of 7.79 (95\% CI 1.02-59.09, p = 0.048) of ischemic type compared to hemorrhagic type in the Cox regression analysis for perioperative stroke within 30 days of bypass surgery in 148 patients with symptomatic MMD (Table 7). Based on a study of 246 bypass surgeries performed among 165 adult MMD patients, Hyun et al. identified the following factors as being associated with perioperative ischemic complications: multiple ischemic episodes, the presence of a preoperative low-density area on CT, and a high signal intensity on diffusion MRI. ${ }^{19}$ Therefore, studies should focus on examining perioperative ischemic events to assess the efficacy and safety of bypass surgery in adult patients with ischemic MMD.

Japanese authors have emphasized the importance of direct bypass, ${ }^{17,29}$ in particular, the JAM trial suggested the preventive effect of direct bypass against bleeding in hemorrhagic MMD. ${ }^{29}$ However, our results showed no difference between direct bypass and indirect bypass for stroke recurrence and death (Fig. 4). In a recent systematic review, postoperative stroke rates between direct bypass and indirect bypass in MMD patients were similar. ${ }^{23}$ In regard to efficacy and safety, which surgical method is better remains uncertain, although direct bypass may offer better angiographic revascularization and a better outcome in adult MMD. . $^{1,310,14,22}$

A recent nationwide, population-based study of adult patients treated with bypass surgery for mortality reduction did not show significant differences in disease course from patients in the medical treatment group, ${ }^{2}$ and similar results were also observed in our Kaplan-Meier analysis (Fig. 3). While the JAM trial and several observational studies have shown functional improvement following 

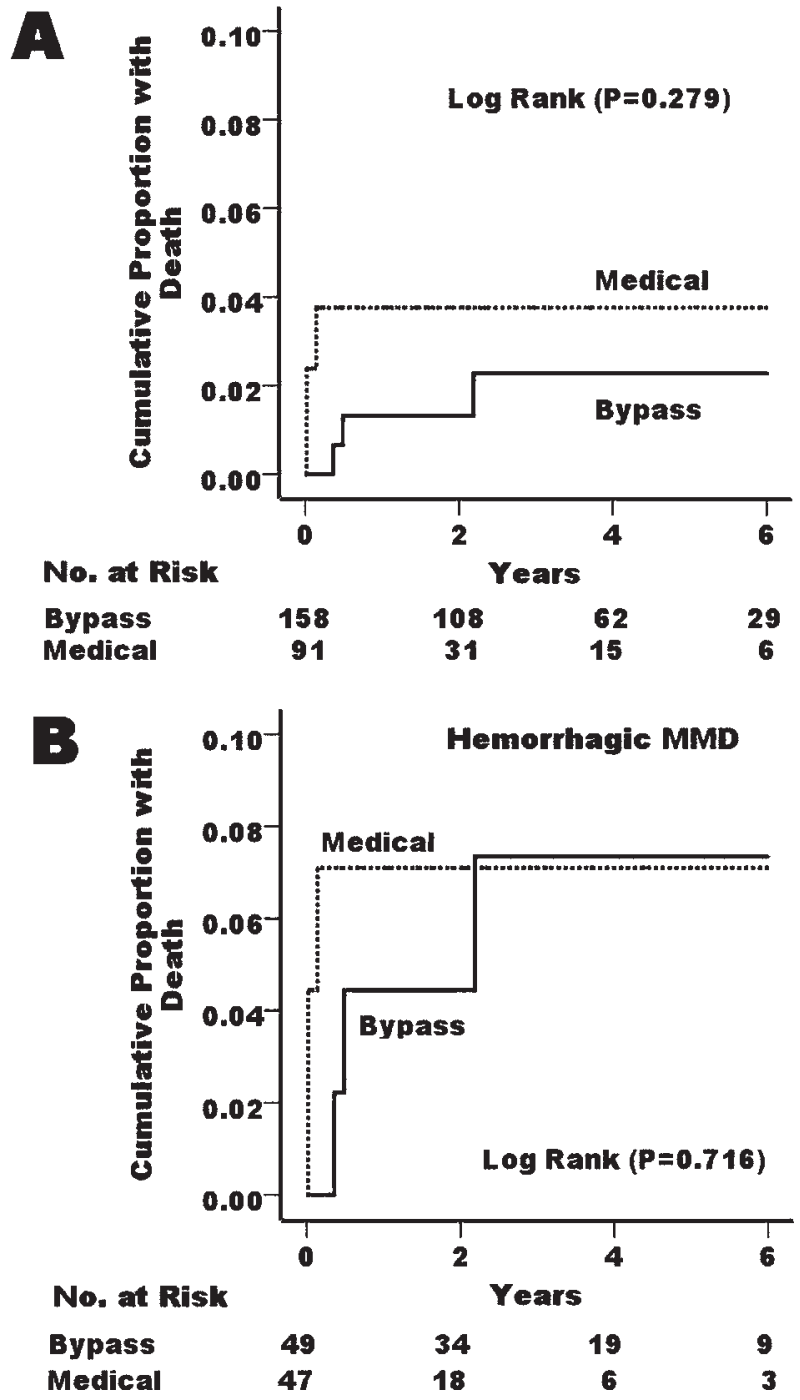

FIG. 3. Kaplan-Meier cumulative curves for death due to recurrent stroke in 249 adult patients (A) and 96 adult patients with hemorrhagic $\operatorname{MMD}(\mathrm{B})$.

bypass surgery, ${ }^{15,27,29}$ its superiority to medical treatment in terms of functional outcome and mortality is controversial. ${ }^{8,36}$ Interestingly, smoking is a strong predictor of stroke recurrence regardless of the presentation type in adult MMD patients (Tables 4-6). Jo et al. reported smoking as a factor associated with clinical and radiological progression. ${ }^{21}$ Therefore, a further epidemiological study to elucidate the association of smoking and future strokes in MMD patients is necessary.

Our study has several limitations and strengths. First, the retrospective cohort study design introduced selection bias; therefore, this study may be influenced inevitably by unmeasurable confounding such as nonstandardized surgical indication. Second, heterogeneous bypass methods were performed in our study, which could have skewed the bypass surgery data to support the null hypothesis, especially in ischemic MMD. Lastly, baseline hemodynamic studies, including cerebral blood flow and cerebrovascular reserve capacity measurements, were not performed. However, this study is important in that it had a large sam-
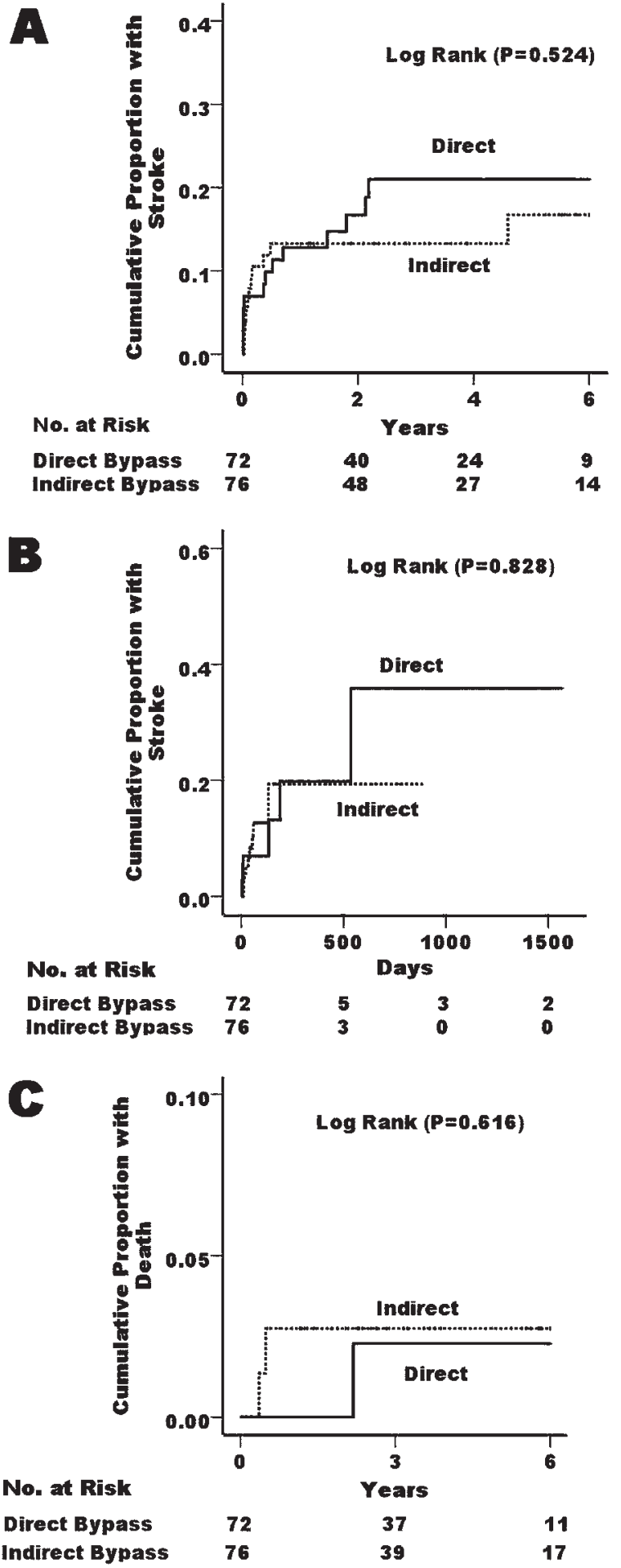

FIG. 4. Kaplan-Meier cumulative curves for stroke recurrence in 148 adult patients treated with direct bypass or indirect bypass surgery over a 6 -year follow-up (A) and within 30 days after bypass surgery (B), and cumulative curves for death according to bypass methods (C).

ple size and was conducted in a real-world setting in adult symptomatic patients with DSA-confirmed MMD. The current study provides guidelines for treatment strategies in adult patients with symptomatic MMD, especially those with ischemic MMD, in the post-JAM trial era. 
TABLE 7. Univariate and multivariate analyses for factors associated with postoperative stroke within 30 days in 148 adults with MMD treated with bypass surgery

\begin{tabular}{|c|c|c|c|c|}
\hline \multirow[b]{2}{*}{ Variable } & \multicolumn{4}{|c|}{ Perioperative Stroke } \\
\hline & Univariate* $^{*}$ & $\begin{array}{c}p \\
\text { Value }\end{array}$ & Multivariate* & $\begin{array}{c}p \\
\text { Value }\end{array}$ \\
\hline \multicolumn{5}{|l|}{ Bypass methods } \\
\hline Indirect & 1.00 (reference) & & & \\
\hline Direct & $0.90(0.35-2.34)$ & 0.828 & & \\
\hline Age (continuous) & $1.01(0.97-1.06)$ & 0.624 & & \\
\hline $\begin{array}{c}\text { Age (>42 yrs, } \\
\text { median) }\end{array}$ & $1.05(0.40-2.76)$ & 0.924 & & \\
\hline Female & $1.01(0.35-2.90)$ & 0.979 & & \\
\hline Family history & NA & & & \\
\hline Hypertension & $2.61(0.95-7.14)$ & 0.062 & & \\
\hline Diabetes & $1.23(0.28-5.40)$ & 0.780 & & \\
\hline Hyperlipidemia & NA & & & \\
\hline Current smoker & NA & & & \\
\hline $\begin{array}{l}\text { Heavy alcohol } \\
\text { drinker }\end{array}$ & NA & & & \\
\hline Heart disease & NA & & & \\
\hline \multicolumn{5}{|l|}{ MMD type } \\
\hline Hemorrhagic & 1.00 (reference) & & & \\
\hline Ischemic & $7.79(1.02-58.09)$ & 0.048 & & \\
\hline $\begin{array}{l}\text { mRS Score 3-5 at } \\
\text { diagnosis }\end{array}$ & $0.69(0.22-2.12)$ & 0.515 & & \\
\hline \multicolumn{5}{|l|}{ Angiographic stage } \\
\hline 1 & 1.00 (reference) & & & \\
\hline 2 & $0.80(0.09-7.04)$ & 0.844 & & \\
\hline 3 & $0.41(0.05-3.58)$ & 0.419 & & \\
\hline 4 & $0.33(0.03-3.76)$ & 0.372 & & \\
\hline 5 & $1.62(0.16-16.31)$ & 0.682 & & \\
\hline 6 & $4.57(0.27-78.60)$ & 0.295 & & \\
\hline Antiplatelet agent & $2.50(0.57-78.60)$ & 0.225 & & \\
\hline \multicolumn{5}{|l|}{$\begin{array}{l}\text { Operated hemi- } \\
\text { sphere }\end{array}$} \\
\hline Unilateral & 1.00 (reference) & & & \\
\hline Bilateral & $1.09(0.38-3.15)$ & 0.869 & & \\
\hline
\end{tabular}

* Presented as $\mathrm{HR}(95 \% \mathrm{Cl})$.

\section{Conclusions}

In this large Korean adult symptomatic MMD cohort, bypass surgery reduced stroke recurrence in hemorrhagic MMD, but its effectiveness on the prevention of new stroke in ischemic MMD is unknown. Regarding mortality reduction, this study failed to show the benefit of bypass surgery compared with medical treatment in adults with MMD. Our findings also suggest that neurosurgeons must recognize that perioperative stroke risk is relatively high during bypass surgery in adult ischemic MMD patients and try to reduce this risk when they perform this surgery. Therefore, a well-controlled prospective randomized investigation is necessary to confirm the effectiveness and safety of bypass surgery, especially in adult ischemic MMD.

\section{Acknowledgments}

We give special thanks to Ye-Kyung Shin and Min-Jung Ham for performing data coding. Statistical analysis was supported by the Catholic Research Coordinating Center of the Korea Health 21 R\&D Project (A070001), Ministry of Health \& Welfare, Republic of Korea.

\section{References}

1. Abla AA, Gandhoke G, Clark JC, Oppenlander ME, Velat GJ, Zabramski JM, et al: Surgical outcomes for moyamoya angiopathy at barrow neurological institute with comparison of adult indirect encephaloduroarteriosynangiosis bypass, adult direct superficial temporal artery-to-middle cerebral artery bypass, and pediatric bypass: 154 revascularization surgeries in 140 affected hemispheres. Neurosurgery 73:430-439, 2013

2. Ahn IM, Park DH, Hann HJ, Kim KH, Kim HJ, Ahn HS: Incidence, prevalence, and survival of moyamoya disease in Korea: a nationwide, population-based study. Stroke 45:1090-1095, 2014

3. Bang JS, Kwon OK, Kim JE, Kang HS, Park H, Cho SY, et al: Quantitative angiographic comparison with the OSIRIS program between the direct and indirect revascularization modalities in adult moyamoya disease. Neurosurgery 70:625-633, 2012

4. Bao XY, Duan L, Li DS, Yang WZ, Sun WJ, Zhang ZS, et al: Clinical features, surgical treatment and long-term outcome in adult patients with Moyamoya disease in China. Cerebrovasc Dis 34:305-313, 2012

5. Cho WS, Kim JE, Kim CH, Ban SP, Kang HS, Son YJ, et al: Long-term outcomes after combined revascularization surgery in adult moyamoya disease. Stroke 45:3025-3031, 2014

6. Choi JU, Kim DS, Kim EY, Lee KC: Natural history of moyamoya disease: comparison of activity of daily living in surgery and non surgery groups. Clin Neurol Neurosurg 99 (Suppl 2):S11-S18, 1997

7. Czabanka M, Peña-Tapia P, Scharf J, Schubert GA, Münch E, Horn P, et al: Characterization of direct and indirect cerebral revascularization for the treatment of European patients with moyamoya disease. Cerebrovasc Dis 32:361-369, 2011

8. Derdeyn CP: Direct bypass reduces the risk of recurrent hemorrhage in moyamoya syndrome, but effect on functional outcome is less certain. Stroke 45:1245-1246, 2014

9. Fujii K, Ikezaki K, Irikura K, Miyasaka Y, Fukui M: The efficacy of bypass surgery for the patients with hemorrhagic moyamoya disease. Clin Neurol Neurosurg 99 (Suppl 2):S194-S195, 1997

10. Fujimura M, Tominaga T: Lessons learned from moyamoya disease: outcome of direct/indirect revascularization surgery for 150 affected hemispheres. Neurol Med Chir (Tokyo) 52:327-332, 2012

11. Fukui M: Guidelines for the diagnosis and treatment of spontaneous occlusion of the circle of Willis ('moyamoya' disease). Research Committee on Spontaneous Occlusion of the Circle of Willis (Moyamoya Disease) of the Ministry of Health and Welfare, Japan. Clin Neurol Neurosurg 99 (Suppl 2):S238-S240, 1997

12. Fukui M, Kono S, Sueishi K, Ikezaki K: Moyamoya disease. Neuropathology 20 Suppl:S61-S64, 2000

13. Fung LW, Thompson D, Ganesan V: Revascularisation surgery for paediatric moyamoya: a review of the literature. Childs Nerv Syst 21:358-364, 2005

14. Gross BA, Du R: Adult moyamoya after revascularization. Acta Neurochir (Wien) 155:247-254, 2013

15. Guzman R, Lee M, Achrol A, Bell-Stephens T, Kelly M, Do $\mathrm{HM}$, et al: Clinical outcome after 450 revascularization procedures for moyamoya disease. Clinical article. J Neurosurg 111:927-935, 2009 
16. Han DH, Kwon OK, Byun BJ, Choi BY, Choi CW, Choi JU, et al: A co-operative study: clinical characteristics of 334 Korean patients with moyamoya disease treated at neurosurgical institutes (1976-1994). Acta Neurochir (Wien) 142:1263-1274, 2000

17. Houkin K, Kamiyama H, Abe H, Takahashi A, Kuroda S: Surgical therapy for adult moyamoya disease. Can surgical revascularization prevent the recurrence of intracerebral hemorrhage? Stroke 27:1342-1346, 1996

18. Hung CC, Tu YK, Su CF, Lin LS, Shih CJ: Epidemiological study of moyamoya disease in Taiwan. Clin Neurol Neurosurg 99 (Suppl 2):S23-S25, 1997

19. Hyun SJ, Kim JS, Hong SC: Prognostic factors associated with perioperative ischemic complications in adult-onset moyamoya disease. Acta Neurochir (Wien) 152:1181-1188, 2010

20. Ikezaki K, Fukui M, Inamura T, Kinukawa N, Wakai K, Ono Y: The current status of the treatment for hemorrhagic type moyamoya disease based on a 1995 nationwide survey in Japan. Clin Neurol Neurosurg 99 (Suppl 2):S183-S186, 1997

21. Jo KI, Yeon JY, Hong SC, Kim JS: Clinical course of asymptomatic adult moyamoya disease. Cerebrovasc Dis 37:94101,2014

22. Kawaguchi S, Okuno S, Sakaki T: Effect of direct arterial bypass on the prevention of future stroke in patients with the hemorrhagic variety of moyamoya disease. J Neurosurg 93:397-401, 2000

23. Kazumata K, Ito M, Tokairin K, Ito Y, Houkin K, Nakayama $\mathrm{N}$, et al: The frequency of postoperative stroke in moyamoya disease following combined revascularization: a single-university series and systematic review. J Neurosurg 121:432440, 2014

24. Kuriyama S, Kusaka Y, Fujimura M, Wakai K, Tamakoshi A, Hashimoto S, et al: Prevalence and clinicoepidemiological features of moyamoya disease in Japan: findings from a nationwide epidemiological survey. Stroke 39:42-47, 2008

25. Kuroda S, Hashimoto N, Yoshimoto T, Iwasaki Y: Radiological findings, clinical course, and outcome in asymptomatic moyamoya disease: results of multicenter survey in Japan. Stroke 38:1430-1435, 2007

26. Liu X, Zhang D, Shuo W, Zhao Y, Wang R, Zhao J: Long term outcome after conservative and surgical treatment of haemorrhagic moyamoya disease. J Neurol Neurosurg Psychiatry 84:258-265, 2013

27. Liu XJ, Zhang D, Wang S, Zhao YL, Teo M, Wang R, et al: Clinical features and long-term outcomes of moyamoya disease: a single-center experience with 528 cases in China. J Neurosurg 122:392-399, 2015

28. Mallory GW, Bower RS, Nwojo ME, Taussky P, Wetjen NM, Varzoni TC, et al: Surgical outcomes and predictors of stroke in a North American white and African American moyamoya population. Neurosurgery 73:984-992, 2013

29. Miyamoto S, Yoshimoto T, Hashimoto N, Okada Y, Tsuji I, Tominaga T, et al: Effects of extracranial-intracranial bypass for patients with hemorrhagic moyamoya disease: results of the Japan Adult Moyamoya Trial. Stroke 45:1415-1421, 2014

30. Nariai T, Matsushima Y, Imae S, Tanaka Y, Ishii K, Senda
M, et al: Severe haemodynamic stress in selected subtypes of patients with moyamoya disease: a positron emission tomography study. J Neurol Neurosurg Psychiatry 76:663-669, 2005

31. Okada Y, Shima T, Nishida M, Yamane K, Yamada T, Yamanaka C: Effectiveness of superficial temporal artery-middle cerebral artery anastomosis in adult moyamoya disease: cerebral hemodynamics and clinical course in ischemic and hemorrhagic varieties. Stroke 29:625-630, 1998

32. Piao R, Oku N, Kitagawa K, Imaizumi M, Matsushita K, Yoshikawa T, et al: Cerebral hemodynamics and metabolism in adult moyamoya disease: comparison of angiographic collateral circulation. Ann Nucl Med 18:115-121, 2004

33. Powers WJ, Clarke WR, Grubb RL Jr, Videen TO, Adams HP Jr, Derdeyn CP: Extracranial-intracranial bypass surgery for stroke prevention in hemodynamic cerebral ischemia: the Carotid Occlusion Surgery Study randomized trial. JAMA 306:1983-1992, 2011 (Erratum in JAMA 306:2672, 2011)

34. Starke RM, Komotar RJ, Connolly ES: Optimal surgical treatment for moyamoya disease in adults: direct versus indirect bypass. Neurosurg Focus 26(4):E8, 2009

35. Starke RM, Komotar RJ, Hickman ZL, Paz YE, Pugliese AG, Otten ML, et al: Clinical features, surgical treatment, and long-term outcome in adult patients with moyamoya disease. Clinical article. J Neurosurg 111:936-942, 2009

36. Sundaram S, Sylaja PN, Menon G, Sudhir J, Jayadevan ER, Sukumaran S, et al: Moyamoya disease: a comparison of long term outcome of conservative and surgical treatment in India. J Neurol Sci 336:99-102, 2014

37. Suzuki J, Takaku A: Cerebrovascular "moyamoya" disease. Disease showing abnormal net-like vessels in base of brain. Arch Neurol 20:288-299, 1969

38. Uchino K, Johnston SC, Becker KJ, Tirschwell DL: Moyamoya disease in Washington State and California. Neurology 65:956-958, 2005

\section{Disclosures}

The authors report no conflict of interest concerning the materials or methods used in this study or the findings specified in this paper.

\section{Author Contributions}

Conception and design: Lee, Jang, Huh, Yang, Park, Ahn, Sung, Han. Acquisition of data: all authors. Analysis and interpretation of data: Lee, Jang. Drafting the article: Jang. Critically revising the article: Lee, Jang. Reviewed submitted version of manuscript: Lee, Jang, Rha, Huh. Approved the final version of the manuscript on behalf of all authors: Lee. Statistical analysis: Lee, Jang. Study supervision: Lee, Rha.

\section{Correspondence}

Kwan-Sung Lee, Department of Neurosurgery, Seoul St. Mary's Hospital, The Catholic University of Korea, College of Medicine, 222 Banpo-Daero, Seocho-Gu, Seoul 06591, Korea. email: nslk@ catholic.ac.kr. 